\section{CPS-383 DRUG PRESCRIPTION IN THE ELDERLY. ARE WE DOING WELL?}

Z Rodríguez Fernández, MA González González, E Gutiérrez Gutiérrez, E Martínez Álvarez*, A Velez Blanco, X Casás Fernández, N Alvarez Núñez, N Ferreras López, C De Castro Avedillo, JC Saez Hortelano, JJ Ortiz De Urbina González. León University Hospital, Hospital Pharmacy, León, Spain

\subsection{6/ejhpharm-2021-eahpconf.215}

Background and importance Inappropriate use of drugs in elderly patients has health consequences for the patient and for the health system.

Aim and objectives The main objective was to review medication in older adults, especially potentially inappropriate medications (PIMs) using the screening tool of older persons' prescriptions (STOPP). The secondary objective was to assess the possibility of detecting prescription errors by the pharmacist in community pharmacies and in primary care, through the use of the electronic prescription.

Material and methods A descriptive cross sectional study was conducted at a tertiary hospital. We included patients aged over 75 years who have been admitted as inpatients to an internal medicine department for 60 days. Study variables were: age, sex, prescription drugs to hospital discharge, electronic prescriptions and PIMs according to the STOPP criteria (2014).

Results 55 patients were selected, with an average age of $84.47 \pm 496$ years and a male/female ratio of 0.89 .437 prescriptions were analysed. Regarding the number of prescribed drugs per patient: $27.27 \%(n=15)$ patients were prescribed $0-$ 4 drugs; 41.82\% ( $n=23)$ 5-9 drugs; and 30.91\% $(n=17) \geq 10$ drugs. Analysing PIMs according to the STOPP criteria (2014): 187 PIMs (42.79\%) were detected. 163 PIMs $(87.17 \%)$ could be detected by electronic prescriptions. In the group of patients with $0-4$ prescribed drugs, $19.25 \% \quad(n=36)$ of PIMs were detected; $42.78 \%(n=80)$ in the group of 5-9 drugs; and $37.97 \%(n=71)$ in patients with $\geq 10$ drugs. The most common PIMs were: concomitant administration of two drugs of the same class $14,97 \%(n=28)$; and any medication prescribed longer than that indicated $12.29 \%(n=23)$.

Conclusion and relevance The results of the study showed a significant number of PPIs and a profile of polymedicated patients ( $\geq 5$ prescribed drugs), associating polypharmacy with an increase in the number of PIMs. Therefore, there is a need to analyse and correct inappropriate medications and discrepancies in medical prescriptions to hospital discharge in our elderly patients. For this, the hospital pharmacist is a key element. In addition, the intervention of pharmacists from pharmacies and health centres who can verify the electronic prescription is possible and can make an important contribution.

\section{REFERENCES AND/OR ACKNOWLEDGEMENTS}

Conflict of interest No conflict of interest

\section{CPS-384 POTENTIAL DRUG RELATED PROBLEMS IN THE TIME OF COVID-19}

J Barceló-Vidal*, N Carballo, M De Antonio-Cuscó, X Fernández-Sala, D Echeverría-Esnal, P Acín, C López-Mula, L Comella-Anaya, E González-Colominas, S Luque, 0 Ferrández. Hospital De Mar, Pharmacy, Barcelona, Spain
Background and importance Drug related problems (DRP) are common among hospitalised patients. During the COVID-19 pandemic, the number of inpatients increased and the pattern of drug use was varied which could lead to a higher number of potential DRP.

Aim and objectives To describe DRP in patients admitted to COVID-19 wards during the COVID-19 pandemic peak.

Material and methods A retrospective observational study was performed in a tertiary university hospital from 21 March to 30 April 2020. Patients included were those admitted to a COVID-19 ward and presenting a DRP (excluding emergency department and critical care units). Computerised physician order entry (CPOE) operates for all hospital beds. Medical prescriptions were revised daily by clinical pharmacists. When a potential DRP was detected, an annotation with a recommendation was made in the patient's medical record. DRP were classified according to the Pharmaceutical Care Network Europe classification.

Abstract 4CPS-384 Table 1

\begin{tabular}{|c|c|c|}
\hline \multicolumn{3}{|l|}{ PRM } \\
\hline \multirow[t]{2}{*}{ Wrong dosage } & Overdose & $90(22.9)$ \\
\hline & Underdose & $38(9.7)$ \\
\hline \multicolumn{2}{|l|}{ Out of protocol } & 78(19.8) \\
\hline \multirow[t]{3}{*}{ Interactions } & Adverse event potentiation & $34(8.7)$ \\
\hline & Induction/inhibition & $15(3.8)$ \\
\hline & Bioavailability alteration & $12(3.1)$ \\
\hline \multicolumn{2}{|c|}{ Prescription error by incorrect use of CPOE } & $41(16.1)$ \\
\hline \multicolumn{2}{|l|}{ Renal impairment } & $25(6.4)$ \\
\hline \multirow[t]{2}{*}{ Indication } & Inexistent drug & $16(4.1)$ \\
\hline & Unnecessary drug & $9(2.3)$ \\
\hline \multicolumn{2}{|l|}{ Other } & $35(8.9)$ \\
\hline \multicolumn{3}{|l|}{ Recommendation } \\
\hline \multirow[t]{3}{*}{ Acceptance rate } & Accepted & $284(72.2)$ \\
\hline & Non-accepted & $60(15.2)$ \\
\hline & Non-evaluable & $49(12.5)$ \\
\hline \multicolumn{3}{|l|}{ Drug involved } \\
\hline$P$ & Hydroxychloroquine & $90(22.9)$ \\
\hline \multirow[t]{5}{*}{ J } & Ceftriaxone & $65(16.5)$ \\
\hline & Azithromycin & $27(6.9)$ \\
\hline & Lopinavir/ritonavir & $6(1.5)$ \\
\hline & Dolutegravir & $1(0.02)$ \\
\hline & Other & $14(3.6)$ \\
\hline \multirow[t]{5}{*}{ A } & Calcifediol & $34(8.6)$ \\
\hline & Vitamin B & $2(0.5)$ \\
\hline & Saccharomyces boulardii & $9(2.3)$ \\
\hline & Calcium carbonate & $4(1.0)$ \\
\hline & Potassium & $4(1.0)$ \\
\hline \multirow[t]{2}{*}{$\mathrm{H}$} & Dexamethasone & $25(6.4)$ \\
\hline & Methylprednisolone & $4(1.0)$ \\
\hline \multirow[t]{4}{*}{ B } & Enoxaparin & $15(3.8)$ \\
\hline & AAS & $2(0.05)$ \\
\hline & Apixaban & $2(0.05)$ \\
\hline & Acenocumarol & $1(0.02)$ \\
\hline $\mathrm{R}$ & Respiratory system & $13(3.3)$ \\
\hline \multirow[t]{2}{*}{ C } & Lipid modifying agents & $10(2.5)$ \\
\hline & Diuretics & $7(1.8)$ \\
\hline $\mathrm{N}$ & Psychoanaleptics & $7(1.8)$ \\
\hline V & Various & $8(2.0)$ \\
\hline \multicolumn{2}{|l|}{ Other } & $43(10.9)$ \\
\hline
\end{tabular}

